was considered (on admission MELD score 24, King's Wilson index 12; on day 10 MELD score 18, King's Wilson index $8)$.

The decision was made to attempt plasma exchange over three days and introduce penicillamine. The patient improved clinically, tolerated penicillamine well and was released subsequently after 34 days of hospitalization and five plasma exchanges overall. Liver synthetic function completely normalized few months later. Genetic analysis of ATP7B gene (H1069Q mutation) confirmed presence of the H1069Q mutation on one allele.

We presented a patient suffering from WD in whom the disease presented acutely with development of liver failure with near-normal values of liver enzymes, mild hyperbilirubinemia, markedly impaired synthetic liver function and Coombs-negative haemolytic anaemia. Acute liver failure in WD may be successfully managed with plasma exchange therapy.

\section{ACUTE BILIARY PANCREATITIS OCCURRING IN THE EARLY POSTPARTUM PERIOD IN A TEENAGE GIRL: A CASE REPORT}

Mario Mašić*, Iva Hojsak, Oleg Jadrešin, Marko Mesić, Sanja Kolaček, Zrinjka Mišak. Children's Hospital Zagreb

\subsection{6/archdischild-2021-europaediatrics.242}

Introduction Acute biliary pancreatitis during early postpartum period in adolescent pregnancy is a rare, but challenging clinical entity in terms of diagnosis and management. We report a case of acute pancreatitis, occurring in early postpartum period in an adolescent patient who had an otherwise uneventful course of pregnancy.

Case Report A 15-year-old patient presented with a complaint of severe upper abdominal pain with propagation to the back and bilious vomiting, 18 days after delivery of a healthy child. Apart from psychiatric treatment due to behavioral disorder, she was healthy, and the pregnancy was uneventful.

The patient was initially examined and admitted to a secondary institution where the diagnosis of acute pancreatitis was established, due to clinical features, elevated amylase values and abdominal ultrasound findings. After primary stabilization, she was transferred to the Intensive Care Unit of our institution. When admitted, the patient was acutely ill, pale, and afebrile, with slow peristalsis and diffuse abdominal pain. Laboratory values of inflammation markers were elevated (CRP $135.4 \mathrm{mg} / \mathrm{l}$, PCT $1.29 \mathrm{ng} / \mathrm{mL}$ ), with significant increase in lipase (2602 U/L) and amylase in serum (666 U/L) and urine (13593 U/L). Abdominal ultrasound showed enlarged, intumescent pancreas, without necrosis or other focal changes, and multiple concrements in gallbladder, without intrahepatic or extrahepatic duct dilatation, the latter confirmed with magnetic resonance cholangiopancreatography. During hospitalization, patient's condition worsened with the development of a pseudocyst $(66 \mathrm{~mm}$ in diameter) in the tail of pancreas, free fluid in the abdomen and extensive bilateral pleural effusions, which was treated with antibiotics (meropenem), octreotide for the first three days and parenteral fluid, electrolyte and nutrition supplementation. After stabilization, the patient was admitted to Department of Gastroenterology. On the 14th day, a severe abdominal pain reoccurred with propagation to the back and vomiting, with an increase in inflammatory markers and amylase and lipase values.

Abdominal ultrasound showed active pancreatitis with necrotic focal changes in the pancreas. The aforementioned deterioration was inflicted by patient's self-initiated and covert ingestion of nuts and some snacks during the night. After stabilization, laparoscopic cholecystectomy was performed and the patient was discharged.

Conclusion Acute pancreatitis must be kept in mind when evaluating patients presenting with abdominal pain after child delivery, with gallstones being the most common etiological factor. Pregnancy and postpartum associated acute biliary pancreatitis usually has a mild-to-moderate clinical course with a favorable outcome, but can present a challenging clinical entity in adolescence, even posing a survival threat.

\section{RESEARCH OF NUTRITIONAL STATUS OF SURGICAL PATIENTS}

Indira I Khusainova, Anna N Zavyalova, Aleksandr Gostimsky, Oleg Lisovsky, Ivan Lisitsa, Valeriya P Novikova*. Federal State budgetary Educational Institution of Higher Education 'St. Petersburg State Pediatric Medical University' of tre Ministry of Healthcare of the Russian Federation

\subsection{6/archdischild-2021-europaediatrics.243}

Relevance Nutritional status (NS) has an effect on adaptation, pathological process severity, recovery rate, therapy effectiveness, hospitalization duration, etc.

Objective Evaluate NS of children being treated in clinical departments.

Materials and Methods nutritional status of 20 children 7 to 17 years old (average age is 13.8 years old) being treated in the surgical department was evaluated. Body weight, height and body mass index (BMI) as well as chest, waist, hips, hip and non-working arm wrist circumferences were studied; centile corridors (CC) were determined. All children have been evaluated for nutritional status using impedancemetry (body composition). All children were questioned using the nutritional status scale created specifically for this research. Statistical analysis, including parametric statistics methods, as well as Spearman's rank correlations was performed using the Statistics 6.1 software.

Results Children with chronic orthopedic pathology requiring repeated surgical intervention as well as children with acute trauma observed in the 2nd surgical department. Nutritional status was estimated according to anthropometric data using relative values (centile corridors). Height: $20 \%$ are tall (4 children in the 6th and the 7 th centile corridors), 30\% (6

children) are in the 3rd centile corridor, and 25\% (5 children) have stunted growth (the 1 st and the 2 nd centile corridors). Weight: 25\% have excess weight (mainly children with acute pathology), 30\% (6 children) have moderate weight deficit (the 3 rd centile corridor), and 15\% (3 children) have acute weight deficit relative to height. Body mass index: $30 \%$ have excess weight and 20\% have acute deficit in nutritional status. Nutritional status was estimated by the method of impedancemetry, which coincided with the data of anthropometry. High reliable $(p<0.05)$ positive correlations between percentage of fat mass, lean mass, active cell mass and total water and the following anthropometric data: centile corridors of body mass and centile corridors of BMI were established.

Conclusions Thus, according to the totality of studies performed, $30 \%$ of children were in normal nutritional status; 
$15 \%$ of children had stunted growth; $10 \%$ of children showed acute protein-energy deficiency of the 1 st grade and 5\% of children showed acute protein-energy deficiency of the $2 \mathrm{nd}$ grade; $15 \%$ of children have chronic protein-energy deficiency of the 1st grade; different grades of excess nutrition and obesity were detected in $25 \%$ of children.

\section{SONOGRAPHIC PICTURE OF THE CHRONIC PANCREATITIS IN AN 8 YEARS OLD CHILD: CLINICAL CASE}

Kirill Valyalov*, Elena Kaytukova, Elena Komarova, Nato Vashakmadze, Leyla NamazovaBaranova. The Research Institute of Pediatrics and Child Health of the Central Clinical Hospital of the Russian Academy of Sciences of the Ministry of Science and Higher Education of the Russian Federation

\subsection{6/archdischild-2021-europaediatrics.244}

Children's chronic pancreatitis is a casuistic rarity; it is an inflammatory and degenerative disease of the pancreas, which can be associated as a reaction to any pathological process or be of a hereditary nature. Chronic pancreatitis, as a rule, is asymptomatic or with unexpressed clinical signs, and can lead to acute pancreatitis.

An 8 years old boy turned to the department with complains on intermittent cramp-like pain in the abdomen during a year, mostly after meal, on the rare liquid stool or it was absence during several days. The pain passed off on its own or was relieved with antacids. Last year at the other clinic the ultrasound examination of the abdominal cavity organs was conducted. Any pathological changes of the internal organs were not detected. Biochemical blood test was not conducted; the symptomatic therapy was prescribed by attending medical doctor.

Results The ultrasound examination of the hepatobiliary system organs was conducted. Significant changes of the pancreas gland were detected: increases sizes, uneven counters, parenchyma was inhomogeneous due to different tissue areas of incorrect shape, of decreased and increased echoicity; multiple hyperechoic inclusions with sizes up to $1,5-8,0 \mathrm{~mm}$ with acoustic shadow were visualized in the tail's region. The Wirsung's duct was expanded among along its entire length up to $5,0 \mathrm{~mm}$ with a wavy course, calculi and clots of secretion were detected in the lumen. No mass lesions were detected. Multiple lymph nodes wit sizes up to $1 \mathrm{~cm}$ of normal shape with preserved architectonics were detected in the part of the pancreas head. No changes of peripancreatic mass were detected.

Moderate quantity of free liquid was visualized in the abdominal cavity.

Sonographic data correspond to the chronic pancreatitis. Conducted laboratory examinations showed the increase of alpha-amylase up to $624 \mathrm{u} / 1$ and lipases up to $202 \mathrm{u} / 1$, that confirmed the inflammatory process in the pancreas gland, the results of computed tomography of the abdominal cavity organs with a contrast enhancement of volumetric formations and foci of abnormal uptake of contrast medium were not detected. The patient was referred to the surgeon's consultation to solve the issue of the further treatment tactics.

Conclusion Preserved abdominal pain can suggest the chronicity of inflammatory process in the pancreas gland, the patient is recommended to conduct the ultrasound examination not rare than once in 6 months due to the clinical data.

\section{CLINICAL AND IMMUNOGENETICAL CHARACTERISTIC OF CELIAC DISEASE IN PAEDIATRIC PATIENTS FROM SINGLE TERTIARY CENTRE}

Jana Ivančić ${ }^{*}$ Renata Žunec, Zorana Grubić, Mirna Aničić, Lana Omerza, Duška TješićDrinković, Jurica Vuković, Irena Senečić-Čala. Zagreb School of Medicine, University Hospital Centre Zagreb, Zagreb, Croatia

\subsection{6/archdischild-2021-europaediatrics.245}

Celiac disease (CD) is an immune-mediated systemic inflammatory disease triggered by gluten ingestion in genetically predisposed individuals with a wide spectrum of sings and symptoms. Genetic predisposition is strongly associated with HLA-DQ2 and DQ8 heterodimers (HD), also related to a higher risk of occurrence in other autoimmune conditions.

Objective To investigate clinical and immunogenetic characteristics of patients with celiac disease diagnosed in a tertiary center.

The data collected retrospectively from medical records from 2008.-2018.

included age at the time of diagnosis, clinical presentation, results of specific antibody testing, small bowel biopsy and HLA typing A total of 55 patients were enrolled, 29 females and 26 males (1.12:1), mean age $11.7 \pm 4.62$ years. A classical form of $\mathrm{CD}$ (diarrhea, abdominal distension, failure to thrive) was found in 22 patients, (mean age at diagnosis 3.41 $\pm 3,23$ yrs, median 1,88). 18 patients had an atypical, extraintestinal presentation (mean age $9.31 \pm 3,86 \mathrm{yrs}$, and median 9 yrs).

The asymptomatic disease was found in 15 patients, mainly with diabetes type I (T1D), (mean age 9,27 $\pm 2,67 \mathrm{yrs}$, median 9). Out of 39 patients who underwent HLA typing, 36 patients were positive for either single or double dose of DQ2.5 or DQ8 HD. Among three HD negative patients, one was gene

DQB1*02 double dose positive (HD DQ2.2 homozygous), one was positive for DQA gene of DQ2.5 HD, while the third one was negative for all DQA or DQB genes from CD predisposing DQ HDs. A total of 17 patients positive at very high CD risk according to the presence of either DQ2.5 in double dose or heterozygous DQ2.5/DQ8 genotype or double DQB1*02 gene dose (DQ2.5/DQ2.2 genotype) were mainly asymptomatic. Furthermore, among 11 patients positive for DQ2.5/DQ2 or DQ2.5/DQ8 genotype (double HD dose), 7 were also T1D patients, while in the remaining 28 DQ2.5 or DQ8 heterozygous and HD negative patients, only 4 were diagnosed with T1D; the observed difference was statistically significant $(p=0,004)$.

In our group of $\mathrm{CD}$ patients, clinical presentation related to age was similar to the literature data, with typical gastrointestinal manifestation in the youngest patients. Considering HLA-DQ heterodimer frequency, our patients had a similar distribution to other European and non-European patients with CD. However, since the majority of children with either DQ2.5 homozygous genotype or DQ2.5/DQ8 heterozygous genotype were $\mathrm{CD}$ asymptomatic patients primarily diagnosed with T1D, we would like to emphasize the importance of screening for celiac disease in this group of patients. 